\title{
Experimental investigation and Monte Carlo simulations of radionuclide production inside the Uranium spallation target QUINTA irradiated with a 660-MeV proton beam
}

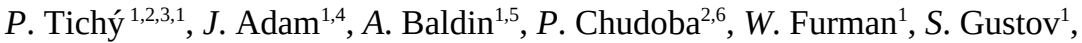 \\ J. Khushvaktov ${ }^{1}, I$. Mar'in ${ }^{1}, A$. Solnyshkin ${ }^{1}$, M. Suchopár ${ }^{2}, J$. Svoboda ${ }^{1,4}$, \\ $S$. Tyutyunnikov ${ }^{1}, R$. Vespalec ${ }^{1,3}, J$. Vrzalová ${ }^{1,2}, V$. Wagner ${ }^{2}, L$. Závorka ${ }^{1}$, and M. Zeman ${ }^{1,4}$ \\ ${ }^{1}$ Joint Institute for Nuclear Research, Joliot-Curie 6, 141980 Dubna, Russia \\ ${ }^{2}$ Nuclear Physics Institute of the ASCR PRI, Hlavní 130, 25068 Řež, Czech Republic \\ ${ }^{3}$ Faculty of Nuclear Sciences and Physical Engineering, Czech Technical University in Prague, \\ Břehová 7, 11519 Prague 1, Czech Republic \\ ${ }^{4}$ Faculty of Electrical Engineering and Communication, Brno University of Technology, \\ Technická 3058/10, 61600 Brno, Czech Republic \\ ${ }^{5}$ Institute for Advanced Studies "OMEGA", Universitetskaya 19, 141980 Dubna, Russia \\ ${ }^{6}$ Faculty of Mathematics and Physics, Charles University, Ke Karlovu 3, 12116 Prague 2, Czech \\ Republic
}

\begin{abstract}
The accelerator-Driven-System (ADS) is very important to study the neutron field and radionuclide production inside simple-geometry uranium subcritical setups irradiated with high energy particle beams. A subcritical setup QUINTA was irradiated with the $660-\mathrm{MeV}$ proton beam from Phasotron accelerator at the Joint Institute for Nuclear Research (JINR). The radionuclide production in the region along the beam axis was investigated by the activation technique. The aim was to compare $(\mathrm{n}, \mathrm{x})$ with $(\mathrm{p}, \mathrm{x})$ reactions using activation detectors of ${ }^{59} \mathrm{Co}$ and ${ }^{\mathrm{nat}} \mathrm{Pb}$, and compare experimental results with the calculated results using Monte Carlo simulation code MCNPX 2.7.
\end{abstract}

\section{Introduction}

ADS studies at JINR have a long tradition. Experiments are usually performed on simple experimental setups (consisting of a uranium or lead spallation target and uranium subcritical blanket) which are irradiated with high energy proton, deuteron or carbon beams from the Phasotron [1] or Nuclotron [2] accelerators. The primary beam impinges the target and a high-intensity neutron field is generated inside the setup by spallation and fission reactions. The first experiments were carried out with the GAMMA-2 setup [3], followed by GAMMA-3 [4] and Energy plus Transmutation (E+T) [5]. The latest irradiations were carried out with the QUINTA setup [6-8] and experiment preparations with the newest

\footnotetext{
${ }^{1}$ e-mail: tichy@jinr.ru
} 
BURAN setup [9] are in progress nowadays. In this paper, radionuclide production along the beam passage was investigated by gamma spectrometry methods. Activation foils of ${ }^{59} \mathrm{Co}$ were used for its sensitivity both to neutrons and protons, and foils of ${ }^{\text {nat }} \mathrm{Pb}$ were used because the production of $\mathrm{Bi}$ is induced only with protons. Neutron-induced generation of $\mathrm{Bi}$ isotopes from $\mathrm{Pb}$ is not possible.

Our aim was also the validation of Monte Carlo codes, which is an important part of the ADS research. The codes need to be validated because they are significant for ADS and fast nuclear reactor Research \& Development.

Experiments with the QUINTA setup fluently follow the E+T setup experiments [5, 1015]. The E+T experiments seem to be appropriate for Monte Carlo simulation benchmarks. Good agreement between the experimental and simulated data was reached in the last 4$\mathrm{GeV}$ deuteron beam irradiation [10]. Differences for higher energies are possible to improve the codes. In the previous $\mathrm{E}+\mathrm{T}$ experiments, the agreement is also good. Investigations of few discrepancies are under way and overall systematics of the $\mathrm{E}+\mathrm{T}$ experiments is supposed to be prepared.

\section{Materials and methods}

\subsection{Experimental setup}

The setup QUINTA (see Fig. 1) consists of four identical hexagonal sections and one additional section which differs from the others by an entrance beam channel of $80 \mathrm{~mm}$ in diameter in the centre. The sections are of $284 \mathrm{~mm}$ in diameter and they are composed of natural uranium rods of $36 \mathrm{~mm}$ in diameter, $104 \mathrm{~mm}$ in length and $1.72 \mathrm{~kg}$ in weight. The total mass of uranium is $512 \mathrm{~kg}$. Activation detectors are fixed to four aluminium detector holders between the sections in air gaps of $17 \mathrm{~mm}$ thick and two holders in the front and back part of QUINTA. The setup is shielded with a lead shielding of $100 \mathrm{~mm}$ thick with a beam window of $150 \times 150 \mathrm{~mm}^{2}$.

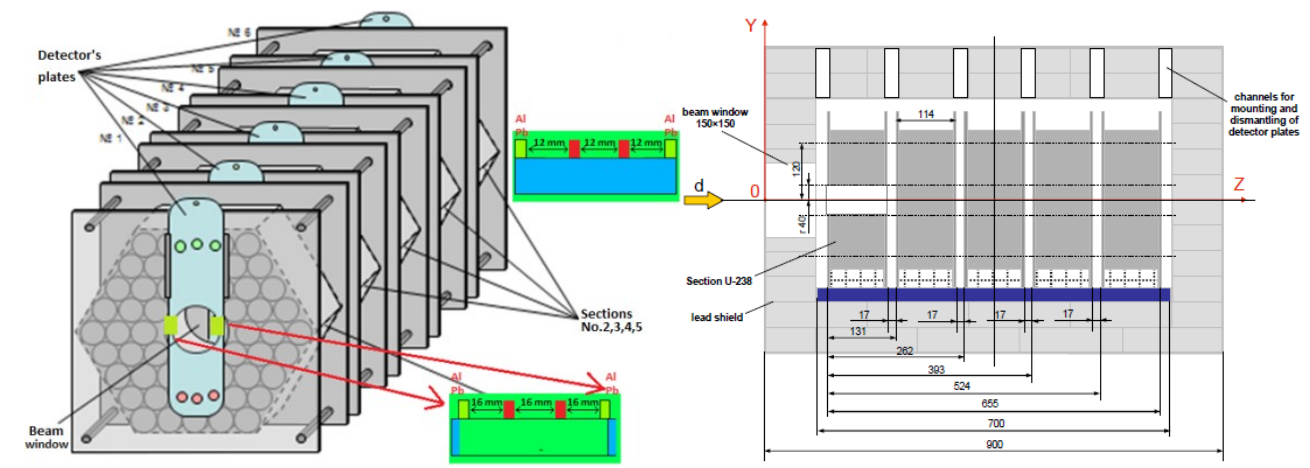

Figure 1. Experimental setup QUINTA [16] and arrangement of cobalt and lead activation detectors. The detectors were positioned on the edges of the aluminium holders. Width of the very front holder is $84 \mathrm{~mm}$. Width of the other holders is $68 \mathrm{~mm}$ thick. Red rectangles symbolize foil placements of the CC experiment (see section 2.3). Distance between the foils on the front holder and the edge of the holder is $2 \mathrm{~mm}$. Beam enters the setup through the beam window. Dimensions are given in mm 


\subsection{Data analysis}

We irradiated the setup QUINTA with the 660-MeV proton beam from the Phasotron accelerator. The advantage of Phasotron is that it can deliver a high intensity beam of $10^{13}$ protons $\cdot \mathrm{sec}^{-1}$. The number of source particles impinging the setup is determined with aluminium activation foils using the monitoring reaction ${ }^{27} \mathrm{Al}(\mathrm{p}, 3 \mathrm{pn})^{24} \mathrm{Na}[16]$.

Gamma spectra of the activation samples are measured with HPGe detectors, acquired by the Genie 2000 software (CANBERRA detectors) [17] or Maestro-32 (ORTEC detectors) [18] and analysed by the DEIMOS32 program [19]. Radionuclide production is analysed with an in-house gamma spectrometry package [20]. Radionuclide production is characterised by a quantity called Reaction Rate (R) which represents a number of nuclei generated in the activation foil normalised to a primary beam particle and the number of atoms of the activation foil. During the analysis, the necessary spectrometry corrections have been used. For more details about the corrections and $\mathrm{R}$ determination see [21, 22].

\subsection{Experiment}

We carried out three irradiations of QUINTA. The first experiment took place in November 2015, the second one in December 2016 and the third one in June 2017. The ${ }^{59} \mathrm{Co}$ and ${ }^{\text {nat }} \mathrm{Pb}$ activation foils were positioned on the left and right sides of the QUINTA aluminium holders as shown in Fig. 1. The difference between these three experiments is that in December 2016, the samples were put even closer to the central axis (exactly between sample positions of the other two experiments) and that the June 2017 irradiation was performed without the lead shielding around the setup. Below, let us distinguish the November 2015 experiment by a symbol "UA" (usual arrangement), the December 2016 experiment - by a symbol "CC" (closer to centre) and the June 2017 experiment - by a symbol "WS" (without shielding).

The field of primary and secondary particles is expected to be different on the left and right sides of the aluminium holders because QUINTA is rotated 2 degrees with respect to the central beam axis as shown in Fig. 2. Another reason why the radionuclide production on the left and right side detectors can be different is that the proton beam adjustment is not the same during each experiment. Beam characteristics like coordinates of the centre of the primary beam entrance into the target (see Fig. 2) and full width at half maximum (FWHM) of the 2D Gaussian profile on the setup entrance of the UA and CC experiments are given in Tab. 1. The characteristics were measured by ionization chambers and they were also used as input parameters of the simulations. Unfortunately, the parameters are missing for the WS experiment due to dosimetry difficulties. 

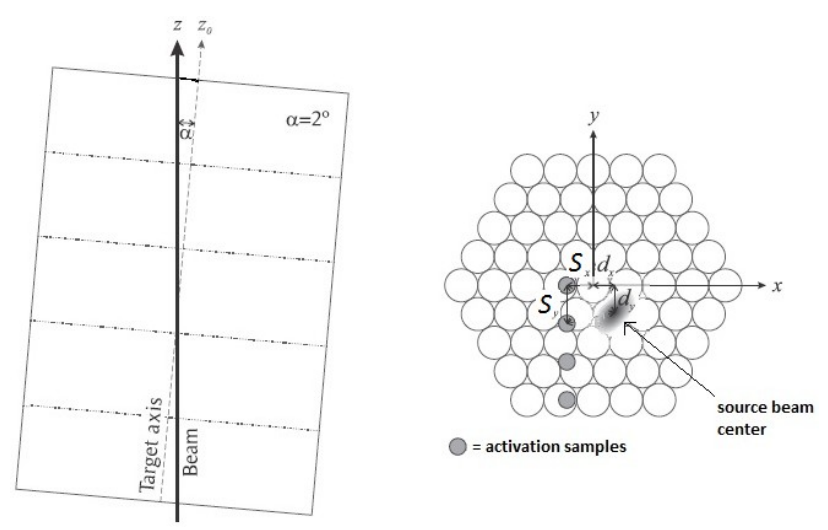

Figure 2. The 2-degree QUINTA rotation from the source beam axis (top QUINTA view, left) and shift of the primary beam centre depending on beam settings (front QUINTA view, right). The symbol $\alpha$ represents the deviation between the beam axis $\mathrm{z}$ and target axis $\mathrm{z}_{0} . \mathrm{S}_{\mathrm{x}}$ and $\mathrm{S}_{\mathrm{y}}$ are the coordinates of an activation sample position on the horizontal axis $\mathrm{x}$ and vertical axis $\mathrm{y} \cdot \mathrm{d}_{\mathrm{x}}$ and $\mathrm{d}_{\mathrm{y}}$ are coordinates of the beam centre on the $\mathrm{x}$ and $\mathrm{y}$ axes. The rotation is mostly made because of the maximal use of the source beam [16, 23]

Table 1. Beam characteristics of the UA and CC experiments at the beam entrance into QUINTA. $d_{x}$ and $d_{y}$ are coordinates of the beam centre position and $\mathrm{FWHM}_{\mathrm{x}}$ and $\mathrm{FWHM}_{\mathrm{y}}$ are full energy widths at half maximum of the 2D beam Gaussian profile

\begin{tabular}{|c|c|c|c|c|}
\hline EXPERIMENT & $\mathrm{d}_{\mathrm{x}}, \mathrm{cm}$ & $\mathrm{d}_{\mathrm{y}}, \mathrm{cm}$ & FWHM $_{\mathrm{x}}, \mathrm{cm}$ & FWHM $_{\mathrm{y}}, \mathrm{cm}$ \\
\hline $\mathrm{UA}$ & 1.31 & 0.76 & 3.40 & 3.97 \\
\hline $\mathrm{CC}$ & 1.40 & 0.67 & 3.11 & 3.60 \\
\hline
\end{tabular}

Dimensions of the ${ }^{59} \mathrm{Co}$ activation detectors were $8 \times 25 \mathrm{~mm}$ and ${ }^{\text {nat }} \mathrm{Pb}$ detectors $8 \times 20$ $\mathrm{mm}$ in all of the three experiments. Weights of the ${ }^{59} \mathrm{Co}$ detectors were about $1.86 \mathrm{~g}$ in all of the three experiments. Weights of the ${ }^{\text {nat }} \mathrm{Pb}$ detectors were about $0.51 \mathrm{~g}$ in the UA experiment, and about $2.80 \mathrm{~g}$ in the CC and WS experiments. Reactions ${ }^{59} \mathrm{Co}(\mathrm{n}, 3 \mathrm{n}){ }^{57} \mathrm{Co}$, ${ }^{59} \mathrm{Co}(\mathrm{n}, 2 \mathrm{n}){ }^{58} \mathrm{Co},{ }^{59} \mathrm{Co}(\mathrm{n}, \mathrm{\gamma}){ }^{60} \mathrm{Co},{ }^{59} \mathrm{Co}(\mathrm{p}, \mathrm{p} 2 \mathrm{n}){ }^{57} \mathrm{Co},{ }^{59} \mathrm{Co}(\mathrm{p}, \mathrm{pn}){ }^{58} \mathrm{Co}$ and ${ }^{\text {nat }} \mathrm{Pb}(\mathrm{p}, \mathrm{x}){ }^{205(206)} \mathrm{Bi}$ were studied. These reactions are convenient to measure their half-lives, gamma intensities and energies.

\subsection{Simulation}

Monte Carlo simulations in MCNPX 2.7 [24] were performed. A detailed model of the QUINTA setup (Fig. 1), including the lead shielding, was prepared. Activation detectors were defined exactly in the same positions as in the real experiments. Neutron and proton fluxes inside the cobalt foils, and proton flux inside the lead foils were calculated. The cross section data library ENDF/B-VII.1 [25] and the intranuclear cascade physics model INCL4.2 [26] and the fission-evaporation ABLA-KHSv3p [27, 28] model were used. Reaction rates of the radionuclides $\mathrm{R}_{\text {sim }}$ under study were calculated by convolutions of the 
flux and cross sections given by TALYS 1.6 [29]. For more information about the convolutions see [30].

\section{Results and discussion}

Representative results of the Phasotron experiments are given and discussed. Both statistical and systematic uncertainties of the experimental values are given. Statistical uncertainties of the simulations have been usually kept below $2 \%$.

In Fig. 3, there are no major differences between the UA and WS experiments. The seen differences are primarily caused due to different beam parameters (coordinates and FWHM). Effects of the lead shielding proved to be negligible for threshold reactions (threshold of the ${ }^{59} \mathrm{Co}(\mathrm{n}, 3 \mathrm{n}){ }^{57} \mathrm{Co}$ reaction is $\left.10.6 \mathrm{MeV}\right)$. The shielding effect is more important for non-threshold reactions, like (n,y), as shown in Fig. 4, where one can notice clearly greater values of ${ }^{60} \mathrm{Co}$ production for the UA experiment. Most of low energy neutrons return to the setup after moderation in the case of the lead shielding. In Fig. 3, there are much greater values of reaction rates for the CC experiment compared to the samples on the left side. It means that the samples on the right side were, probably, hit more by the primary proton beam due to the beam centre position and 2-degree QUINTA rotation. This is also confirmed by ${ }^{58} \mathrm{Co}$ and ${ }^{206} \mathrm{Bi}$ production in Fig. 4 where reaction rate ratios of the CC experiment for the left and right sides are much below the value of 1 .

Agreement between the experimental and simulated reaction rates in Fig. 5 is not bad for the left and right sides of the ${ }^{57} \mathrm{Co}$ production in the UA experiment (the mean value 1.68 for the left side is influenced by the first position which is often problematic because of the beam setting sensitivity). In the CC experiment, a good agreement was reached for the first four points on the right side. The situation on the left side seems to get better with a greater longitudinal distance.

In Fig. 5, the disagreement for the ${ }^{205} \mathrm{Bi}$ and ${ }^{206} \mathrm{Bi}$ production on the right side of the $\mathrm{CC}$ experiment is greater than for the ${ }^{57} \mathrm{Co}$ production. It is because the bismuth production is sensitive just to protons and not to neutrons. The differences between the experiments and simulations can be possibly caused not only by the MCNPX 2.7 code, but also by inaccuracy of accelerator settings.
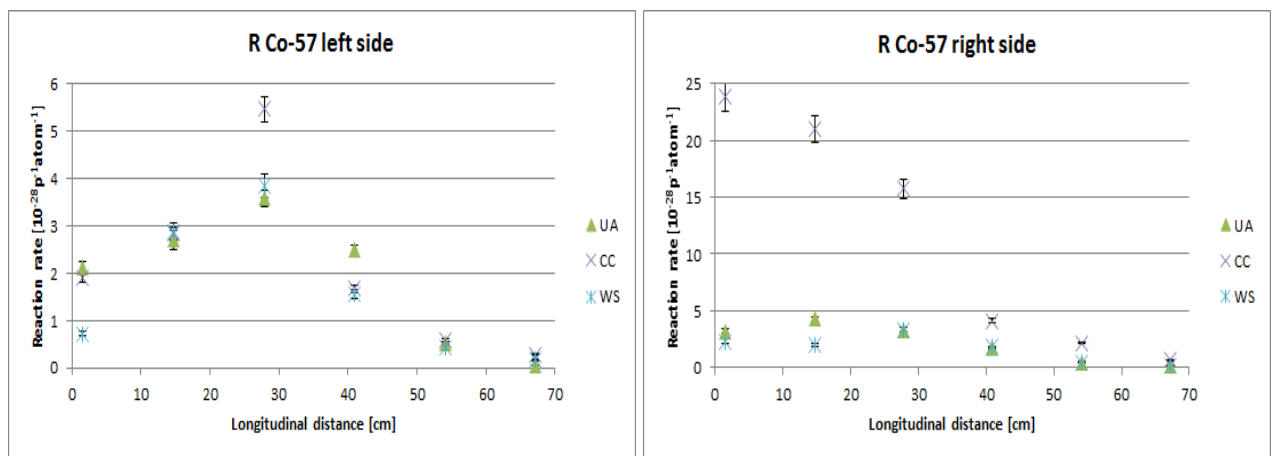

Figure 3. Experimental reaction rates of ${ }^{57} \mathrm{Co}$ production for all the Phasotron experiments for samples on the left and right sides 

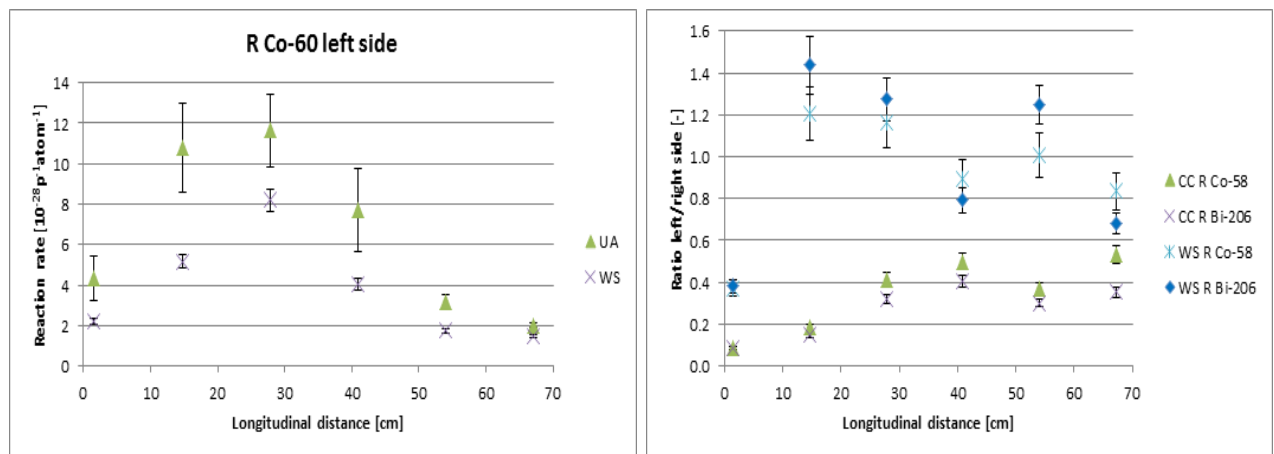

Figure 4. Experimental reaction rates of ${ }^{60} \mathrm{Co}$ production for UA and WS experiments for samples on the left side and left/right side, experimental reaction rate ratios for CC and WS experiments
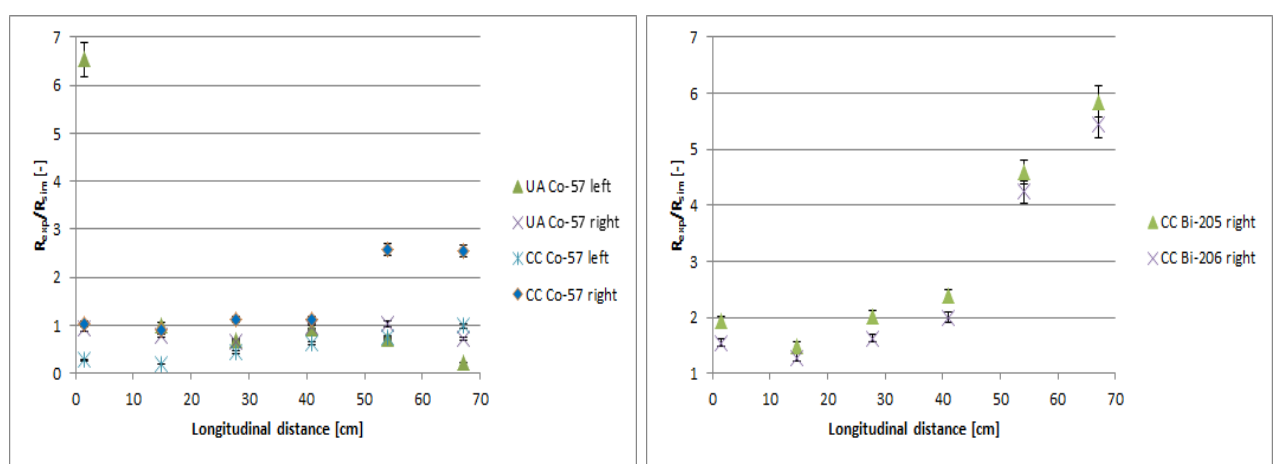

Figure 5. Experimental and simulated reaction rate ratios of ${ }^{57} \mathrm{Co},{ }^{205} \mathrm{Bi}$ and ${ }^{206} \mathrm{Bi}$ production for UA and CC experiments. Mean values are: $<\mathrm{R}_{\exp } / \mathrm{R}_{\text {sim }}>\left({ }^{57} \mathrm{Co}\right.$, UA, left $)=1.68(9),<\mathrm{R}_{\text {exp }} / \mathrm{R}_{\text {sim }}>\left({ }^{57} \mathrm{Co}\right.$, UA, right) $=0.85(4),<\mathrm{R}_{\exp } / \mathrm{R}_{\text {sim }}>{ }^{57} \mathrm{Co}, \mathrm{CC}$, left $)=0.55(3),<\mathrm{R}_{\exp } / \mathrm{R}_{\text {sim }}>\left({ }^{57} \mathrm{Co}, \mathrm{CC}\right.$, right $)=1.54(8),<\mathrm{R}_{\exp } /$ $\mathrm{R}_{\text {sim }}>\left({ }^{205} \mathrm{Bi}\right.$, CC, right $)=3.05(14),<\mathrm{R}_{\text {exp }} / \mathrm{R}_{\text {sim }}>\left({ }^{206} \mathrm{Bi}, \mathrm{CC}\right.$, right $)=2.69(13)$

\section{Conclusion}

Radionuclide production in the setup QUINTA in the region along the primary beam passage has been studied by means of activation technique. Simulations in the MCNPX 2.7 Monte Carlo code have been made and compared with the experimental results.

The effect of the lead QUINTA shielding was assessed (see Figs. 3 and 4). It has been proved that the increase in the reaction rate of the radionuclides produced by non-threshold reactions can be substantial and that for the threshold reactions is negligible.

Radionuclide production in the central region of the QUINTA target along the primary beam passage is very sensitive to the beam settings as shown in Fig. 3. Values of ${ }^{57} \mathrm{Co}$ production for the CC experiment where the activation foils were closer to the central setup axis, have proved to be much higher on the right side than on the left side.

In the central region of the QUINTA target, there are some discrepancies between the experimental and simulated reaction rates (see Fig. 5). A suspicion exists that it can be caused more by inaccurate determination of the coordinates of the proton beam centre on the setup entrance, and the 2-degree setup rotation rather than by the Monte Carlo simulation code MCNPX 2.7. Therefore it is important to study the effect of the beam parameters changes on the simulations. The activation detectors close to the beam help to 
control its parameters and decrease uncertainties of the benchmark using the neutron data obtained from the outer part of the target.

\section{References}

1. A. Dem'yanov et al., PoS (13th Workshop on charged part. accel.) 265-272 (1992)

2. N. Agapov et al., PoS HIAT 2015, 86-88 (2015)

3. J. Adam et al., Nucl. Instrum. Methods Phys. Res. A 562, 741-742 (2009)

4. N. Asquith et al., Radiation Measurement 67, 15-23 (2014)

5. A. Krása et al., Nucl. Instrum. Methods Phys. Res. A 615, 70-77 (2010)

6. L. Závorka et al., Nucl. Instrum. Methods Phys. Res. A 903, 246-261 (2018)

7. J. Adam et al., Applied Radiation and Isotopes 107, 225-233 (2016)

8. P. Tichý et al., PoS INPC 2016, 115 (2016)

9. P. Tichý and M. Suchopár, PoS Baldin ISHEPP XXII, 065 (2014)

10. M. Suchopár et al., Nucl. Instrum. Methods Phys. Res. A 908, 347-360 (2018)

11. O. Svoboda et al., JINR Preprint E15-2011-39 (Dubna, Russia, 2011)

12. O. Svoboda et al., JINR Preprint E15-2009-177 (Dubna, Russia, 2009)

13. A. Krása et al., JINR Preprint E1-2009-195 (Dubna, Russia, 2009)

14. A. Krása et al., JINR Preprint E15-2007-81 (Dubna, Russia, 2007)

15. F. Křížek et al., Czechoslovak Journal of Physics 56, 243 (2006)

16. W. Furman et al., PoS (Baldin ISHEPP XXI) 086 (2012)

17. Genie 2000 V3.3, Operation Manual (Canberra Industries, Inc., 2012)

18. MAESTRO-32 V6.0, User's Manual (Advanced Measurement Technology, Inc., 2006)

19. J. Frána, Journal of Radioanalytical and Nuclear Chemistry 257, 583-587 (2003)

20. J. Adam et al., Measurement Techniques 44, 93-100 (2001)

21. L. Závorka et al., Annals of Nuclear Energy 80, 178-187 (2015)

22. M. Majerle et al., JINR preprint E11-2009-178 (Dubna, Russia, 2009)

23. L. Závorka, Dissertation thesis, Czech Technical University in Prague (2015) (https://ojs.ujf.cas.cz/ wagner/transmutace/diplomky/zavorka/Lukedis.pdf)

24. D. Pelowitz, MCNPX User's Manual V2.7.0, LA-CP-11-00438 (LANL, USA, 2011)

25. M. Chadwick et al., Nuclear Data Sheets 112, 2887-2996 (2011)

26. A. Boudard et al., Physical Review C 66, 1-28 (2002)

27. J. Gaimard and K.H. Schmidt, Nuclear Physics A 531, 709-745 (1991)

28. A. Junghans et al., Nuclear Physics A 629, 635-655 (1998)

29. A. Koning et al., TALYS-1.6, User's Manual (NRG Petten, The Netherlands, 2013)

30. J. Khushvaktov et al., Nucl. Instrum. Methods Phys. Res. B 381, 84-89 (2016) 